\title{
Aspects of Photocatalysis on Semiconductors: Photoelectrocatalysis
}

\author{
Michael Neumann-Spallart*
}

\begin{abstract}
An overview is given of the essential processes governing heterogeneous photocatalysis on semiconductor surfaces. It is shown that the reactions on the surface of the catalyst are of electrochemical nature and can be described using electrochemical principles. This leads to a quantitative description of the processes. In combination with measurements of charge collection yields and Faradaic efficiencies, or product quantum yields, the reaction rates can be calculated when the spectral dependence of the intensity of the light source is known. Examples are given for transition metal oxide semiconductors such as titanium dioxide and tungsten trioxide.
\end{abstract}

Keywords: Oxides · Photocatalysis · Photoelectrochemistry · Semiconductors

Photocatalysis is a topic of vivid research interest, especially as related to its most widely studied potential application, the purification of water from pollutants, mostly of organic origin. Waste water and drinking water are equally appealing targets. Processes involving a photocatalyst in solution, having a chromophore that can be excited will not be dealt with here (quite interesting cases such as the photo-Fenton reaction involving iron complexes in solution might be mentioned ${ }^{[1]}$ ) but, as expressed in the title 'Photocatalysis on Semiconductors', reactions where an interface is involved, the interface between a solid (the semiconductor) and a liquid (in most cases an aqueous solution). As examples for reviews on heterogeneous photocatalysis see the literature. ${ }^{[2,3]}$ Also air purification has

${ }^{*}$ Correspondence: Dr. M. Neumann-Spallart Groupe d'Étude de la Matière Condensée C.N.R.S.

1, place Aristide Briand

F-92195 Meudon CEDEX, France

E-Mail: mns@cnrs-bellevue.fr been studied to some extent. ${ }^{[4]}$ The reactions to be discussed here are mainly oxidative decompositions of organic substances, although systems employing reductive reactions (like $\mathrm{CO}_{2}$ reduction on p-type semiconductors $\left.{ }^{[5]}\right)$ are also of interest.

On semiconductors under illumination with light that can be absorbed, charge carriers (conduction band electrons and valence band holes) are formed. Electron transfer takes place at the interface with the surrounding liquid medium (electrolyte). On an n-type semiconductor, holes lead to oxidative decomposition of (organic) solutes, especially if the holes have a high redox potential, i.e. if the ionization potential of the semiconductor is large. In fact, there are not too many candidates, as stability against anodic dissolution is an important requirement and semiconductors which are not oxides and have a high ionization potential tend to undergo corrosion under illumination. ${ }^{[6]}$ Another aspect of selection is the spectral light sensitivity, the onset of which is determined by the bandgap of the semiconductor, i.e. the distance between conduction band and valence band, being equal to the difference between ionization potential and electron affinity. Together with the other condition, namely having a high ionization potential, this leads to a choice of materials having a large bandgap, which means absorbing only high energy photons i.e. UV light. Examples for such materials are transition metal oxides like titanium dioxide, tungsten trioxide, titanates, and iron oxide. Because of its exceptional stability against photocorrosion, titanium dioxide $\left(\mathrm{TiO}_{2}\right)$ has emerged as the most widely studied photocatalyst, in spite of its photosensitivity being restricted to the UV $(<400 \mathrm{~nm})$. Tungsten trioxide $\left(\mathrm{WO}_{3}\right)$ might be an alternative due to its extended photosensitivity into the visible range (absorption of blue light), making the use of solar light possible.

As the above-mentioned oxides are semiconductors, photocatalysis on large enough particles or bulk material, including thin films in contact with electrolyte, can be understood as an electrochemical process. ${ }^{[7,8]}$ Semiconducting catalysts, immobilized on an electrically conducting surface, can therefore be treated as electrodes, and notions from semiconductor (photo)electrochemistry apply.

In photocatalytic degradation of organic compounds using semiconductor oxides (photocatalysis), most of the studies have been performed with powder suspensions. Processes involving powder suspensions lead to problems arising from the need to separate the photocatalyst from the liquid after completion of the photodegradation reaction. This is especially difficult if the particles are very small. The alternative to a suspension is the use of the photocatalyst in immobilized form, either deposited on larger beads or as films on substrates of various shapes (tubes, plates). Immobilization represents an improvement, but the efficiency of such devices turns out to be as low as it is for powder suspensions due to charge recombination, as their working potential is given by the open circuit condition. Therefore, films supported on conducting substrates, allowing for biasing in an electrochemical reactor and thus avoiding charge recombination as encountered in unbiased systems, are of great interest. 


\section{Electrochemical Characterization}

The immobilization of semiconductor photocatalysts on conducting substrates gives easy access to features to be studied on junctions with liquids, like photopotential, photocurrent and dark current as a function of applied potential. In an n-type semiconductor/electrolyte junction, a gradient of the electrochemical potential is formed which draws holes towards the interface. By applying an external potential, this gradient can be increased to an extent that assures complete separation of all charge carriers (depletion condition), since all majority carriers (electrons) are drawn inside the bulk of the semiconductor and are extracted at the back contact. This is shown in the inset of Fig. 1 where above $0.8 \mathrm{~V}$ the currents under illumination cease to increase and are only limited by the photon flux.

However, at lower potentials, the photocurrent is lower due to carrier recombination. Under illumination in the absence of bias, both charge carriers have to be extracted at the semiconductor/liquid junction. In this case, the working potential, $E_{1}$, is not imposed but auto-established at a value at which anodic currents (photocurrents) and cathodic currents (dark currents), are equal, and the cell current, $i_{\mathrm{scl}}$, is given by the intersection of the individual $i-E$ curves, $i_{\mathrm{ph}}$ and $i_{\mathrm{d}}$ (Fig. 1). Even for a suspension of semiconductor particles $i_{\mathrm{sc}}$ can be estimated from electrochemical analysis of macroscopic electrodes ${ }^{[7]}$ and, again, the larger the cathodic currents, the higher the working current. The reaction of the majority carriers (conduction band electrons) will be oxygen reduction in most cases. Larger dark currents can sometimes be obtained when a noble metal catalyst is deposited on the electrode. Fig. 1 shows the influence of dark currents (oxygen reduction) on local cell currents $\left(i_{\text {sc }}\right)$ on an illuminated $\mathrm{TiO}_{2}$ electrode. In this example, the reduction of oxygen is shown to be enhanced by platinization. Due to the presence of Pt islands, the $\mathrm{O}_{2}$ reduction currents occur at a higher (more than $200 \mathrm{mV}$ ) potential, $E_{2}$, and a higher working current, $i_{\mathrm{sc} 2}$, is obtained. However, the photocurrent is decreased, mainly due to light shading by platinization. As the extent of any oxidation reaction on the electrode(s) (e.g. degradation of solutes) is proportional to $i_{\mathrm{sc}}$, the determination or estimation of $i_{\mathrm{sc}}$ by electrochemical methods is of high relevance for photocatalysis In the absence of other reducible species, oxygen (air saturated or oxygen saturated solution) is the species that is reduced by conduction band electrons. Only if the onset of oxygen reduction is at high enough potentials (more to the right in the present diagram), can a potential be found where the cathodic current, $i_{\mathrm{d}}$, and the anodic current, $i_{\mathrm{ph}}$, are of equal absolute value $\left(\left|i_{\mathrm{d}}\right|=\right.$ $\left.\left|i_{\mathrm{ph}}\right|\right)$, and only if this value is large, large local cell currents, $i_{\text {sc }}$, will flow. (In the absence of dissolved oxygen, reduction cur-

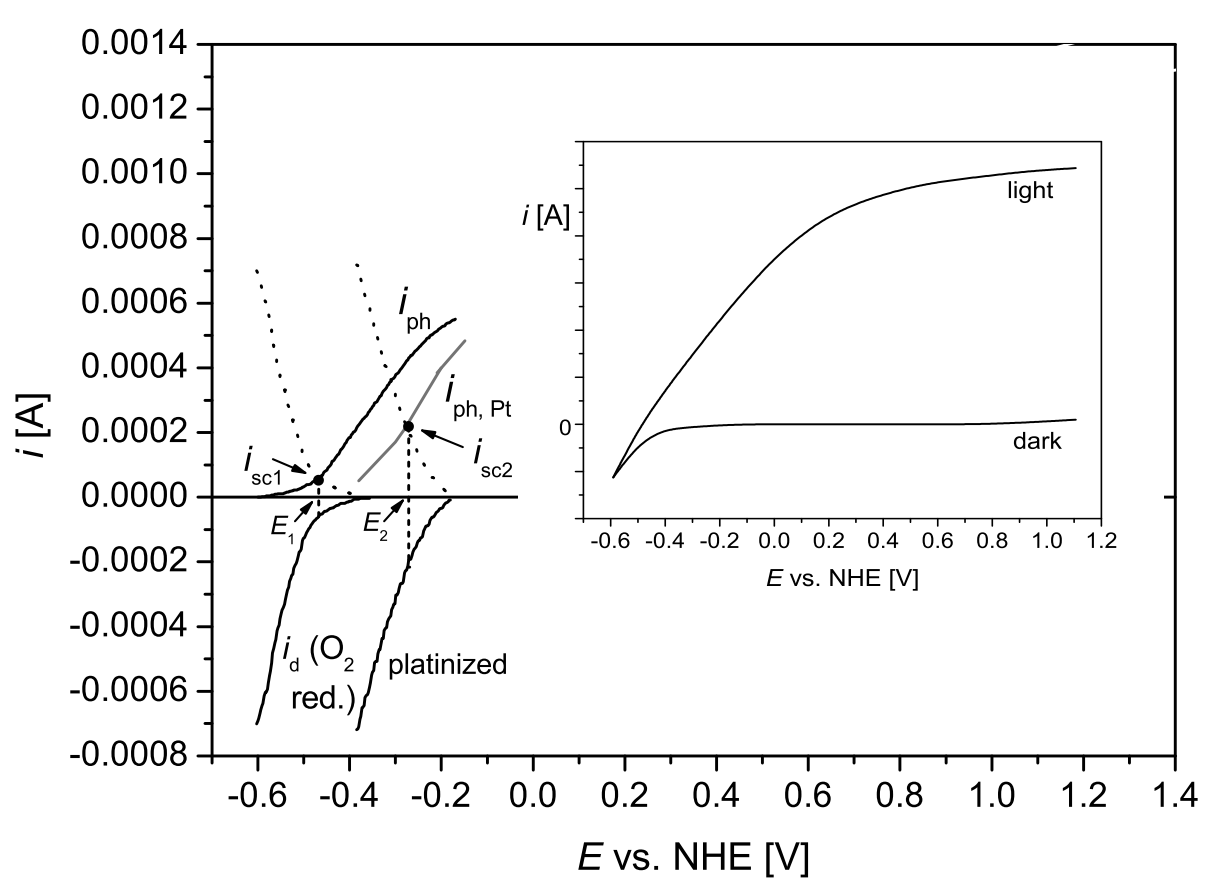

Fig. 1. Influence of dark currents (oxygen reduction) on local cell currents $\left(i_{\mathrm{sc}}\right)$ on an illuminated $\mathrm{TiO}_{2}$ electrode (photocurrent onset region). The electrolyte $(0.1 \mathrm{~N} \mathrm{NaOH})$ was saturated with oxygen and stirred; scan rate: $-0.5 \mathrm{mV} / \mathrm{s},\left(i_{\mathrm{d}} \ldots\right.$ dark currents for $\mathrm{O}_{2}$ reduction, photocurrent $\ldots i_{\text {ph }}=i_{\text {light }}-i_{\text {dark }}$ ). Inset: current - potential curves under light and in the dark over a wide range of potentials. rents (proton reduction) occur only below $-1 \mathrm{~V}$ for the electrode studied in Fig. 1). Oxygen reduction currents and their onset with respect to the onset of photocurrents are not always as favourable as in the case of $\mathrm{TiO}_{2}$. In such a case (for example $\mathrm{WO}_{3}$ ), decomposition reactions will not proceed without the help of electrical bias. The ratio of photocurrents (and therefore the external yield of charge collection or incident photon to electron conversion efficiency, 'IPCE') between the biased condition (full depletion) and the open circuit (unbiased) condition can be several tens and requires an external bias of typically a few hundred $\mathrm{mV}$ between the two electrodes. ${ }^{[7,8]}$ These considerations clearly show the advantage of conducting a photocatalytic reaction under electrical bias.

\section{IPCE}

As the photocurrents depend on light intensity, the efficiency of transforming light flux into electron flow (the IPCE) can be determined at each wavelength using actinometric measurements (absolute determination of light intensity)

$\mathrm{IPCE}=j /(\mathrm{F} \cdot I)$,

with $j=$ photocurrent density, $\mathrm{F}=$ Faraday constant, $I=$ light intensity $\left(\mathrm{E} \mathrm{s}^{-1} \mathrm{~cm}^{-2}\right)$. In the following, IPCE values measured at the plateau region of the photocurrents (sufficiently high applied bias) are taken into consideration, since they relate to the maximum current efficiency for a given electrode preparation.

\section{Dependence on Illumination Source}

Fig. 2 shows the dependence of IPCE on wavelength for a typical $\mathrm{TiO}_{2}$ electrode deposited on an electrically conducting, optically transparent substrate ("conducting glass', F-doped $\mathrm{SnO}_{2}$ ) under illumination through the substrate (SE), and the spectral dependence of the photocurrents, $j$, (curve $\mathrm{i}$ and ii) for two different light sources, a UVA blacklight tube lamp (upper graph) and solar light (lower graph) based on measurement of the spectral intensity, $I$, of the light sources (expressed as current densities; $j_{\mathrm{I}}$ $=I \cdot F)$. The total photocurrent densities, $j_{0}$, for the light sources are given by the area under curve i) and ii), respectively, with $j_{0}=$ $\int \mathrm{IPCE}_{\mathrm{SE}} \cdot J_{\mathrm{I}} \cdot \mathrm{d} \lambda$. For UVA light, $j_{0}=0.000328$ $\mathrm{A} \mathrm{cm}{ }^{-2}$, but also for sunlight, though weak in the UV, appreciable photocurrents can be drawn; $j_{0}=0.000076 \mathrm{~A} \mathrm{~cm}^{-2}$.

As it is desirable to carry out photocatalytic processes with sunlight, it is interesting to compare the spectral sensitivity of semiconductors of different bandgap, 


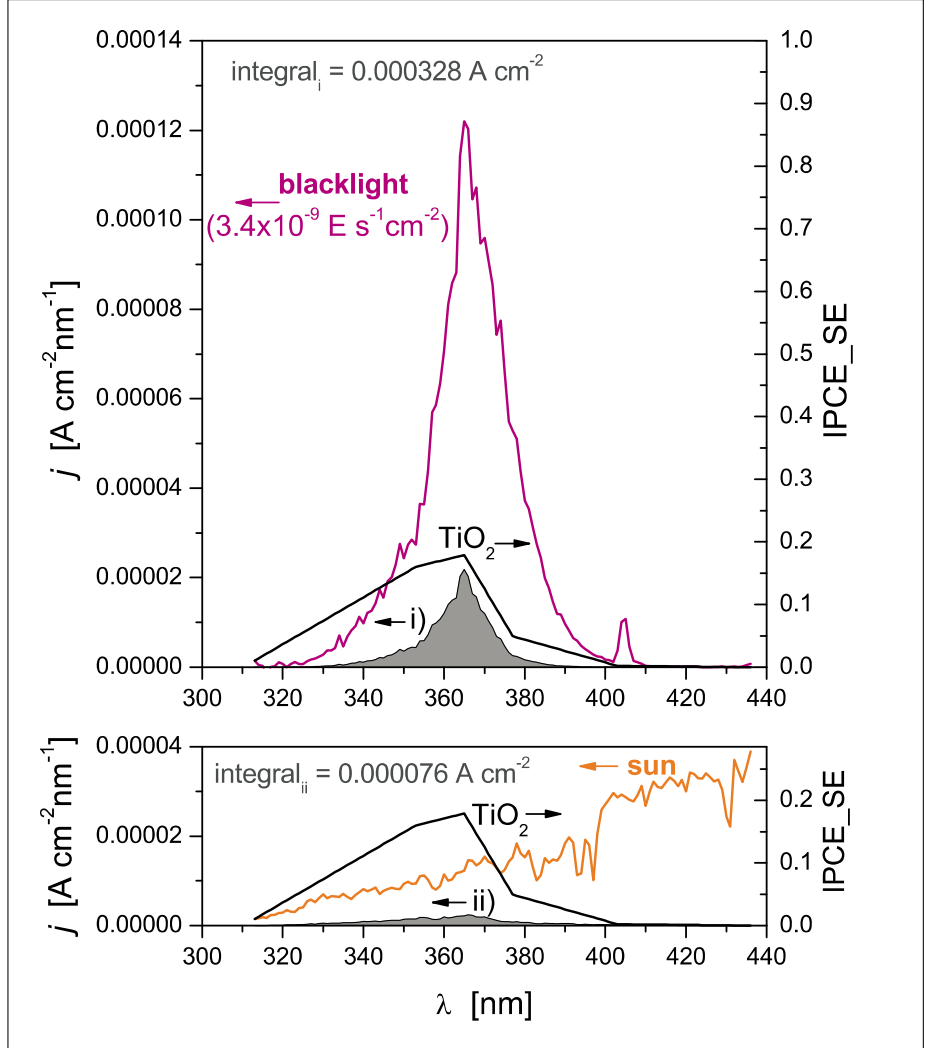

Fig. 2. Influence of light source on the total photocurrent that can be obtained under depletion conditions. Upper part: curve i) ... j.IPCE $\mathrm{SE}_{\mathrm{SE}}$ with the light intensity, $j$, of the blacklight source expressed as current density, lower part: curve ii) ...j.IPCE $\mathrm{SE}_{\mathrm{SE}}$ with the light intensity, $j$, of sunlight expressed as current density.

like $\mathrm{TiO}_{2}$ and $\mathrm{WO}_{3}$. From Fig. 3 it follows that using sunlight as light source, $\mathrm{TiO}_{2}$ is able to deliver a photocurrent density of $0.000076 \mathrm{~A} \mathrm{~cm}^{-2}$ (area under curve i), whereas $\mathrm{WO}_{3}$, the photosensitivity of which extends into the visible, can deliver $0.000793 \mathrm{~A} \mathrm{~cm}^{-2}$ (area under curve ii) i.e. about 10 times more.

\section{Reaction Rates}

Absolute rates of individual electrochemical reactions on the electrode however, cannot be deduced from photocurrent (IPCE) and spectral light intensity measurements alone, as several reactions can take place simultaneously. One of them is oxidation of the solvent (water), being present in large excess. The solvent and the solute (electron donor - a substance which is to be photocatalytically decomposed) therefore compete for the holes (or $\mathrm{OH}$ radicals originating from them). In the absence of an added electron donor the photocurrents correspond to water oxidation, leading to oxygen formation. If a donor is added to the system, part of the photocurrents will correspond to its oxidation, depending on its concentration. This fraction is called the Faradaic efficiency, $f$. The external quantum yield for the oxidation of the donor is given by:

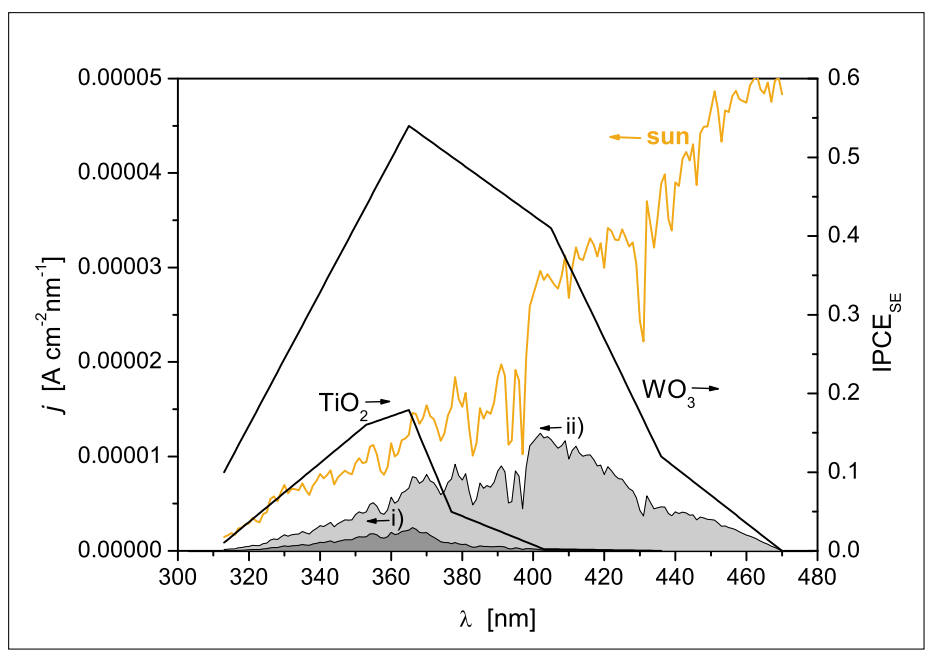

Fig. 3. Comparison of the total photocurrent that can be obtained from

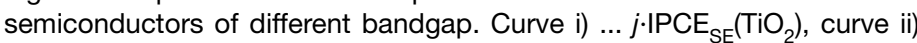
$\ldots j \cdot \mathrm{IPCE}_{\mathrm{SE}}\left(\mathrm{WO}_{3}\right)$. The light intensity, $j$, of sunlight is expressed as current density.

where $m=c V$ in a batch reactor of volume $V$ and electrode area $A$ under light intensity controlled conditions, i.e. when mass transport does not limit the reaction rate. As an example for the dependence of Faradaic efficiency, the degradation of oxalic acid as a model substance in water on a $\mathrm{WO}_{3}$ electrode under illumination was measured and reported. ${ }^{[9]} f$ was obtained from measurements of the $\mathrm{CO}_{2}$ evolution rate, $\mathrm{CO}_{2}$ being the only reaction product (except oxygen) in this system. It was found that $f$ was deviating from the linearity predicted by Eqn. (2) (in fact, two linear sections having a different slope were seen). This is due to the fact that several intermediates, bimolecular reactions, current doubling, and adsorption isotherms come into picture, so that for practical purposes the dependence of $f$ on $c$ has to be measured at several points in the range of concentrations of interest in order to evaluate the decrease of the concentration of an added donor in the course of a degradation experiment precisely. In addition, if bimolecular reactions (of $\mathrm{OH}$ radicals) are to be considered, ${ }^{[10]}$ they are intensity dependent and measurements have to be carried out at the actual light intensity used in the reactor. From the above, it follows that the measurement of IPCE as a function of electrical bias, in combination with $f$, or the direct measurement of product quantum yields as a function of wavelength and the spectral intensity of the light source, allows a full characterisation of a semiconductor layer immobilized on a conducting substrate and the evaluation of the degradation rate in a given reactor. Next to a batch reactor process, a single-pass flowthrough reactor may be used. In this case, the conversion efficiency (solute concentration at the outlet) depends on flow rate. Examples ${ }^{[9]}$ show that the degradation rate, as predicted, can be considerably enhanced when electrical bias is used. 


\section{Effective Surface Area}

The argument that large surface area of a catalyst should help to increase the yield of a photochemical reaction is often found in the literature. Why should that be the case? It is clear that the maximum achievable efficiency depends on the fraction of the photons that is absorbed by the catalyst and on the internal charge collection efficiency which is given by the ratio of separated charge carriers and generated charge carriers. This ratio cannot be larger than 1 and is reached when each absorbed photon leads to the transfer of one electron from a solute to the electrical circuit ('inner' electrical circuit in the case of particles in suspension or 'outer' circuit in the case of an electrochemical cell). Finally, for the desired reaction to be carried out, the Faradaic efficiency is to be taken into account. In the case of a particle suspension, as the working potential, $E_{\mathrm{m}}$, cannot be externally set, the efficiency of charge separation is dictated by the light and dark current potential characteristics of the electrode (see Fig. 1). Therefore, if, for the same amount of light absorbed, this amount is shared in one case by a certain number of particles, and, in another case by more particles (e.g. because the particles are smaller and therefore more transparent or because the concentration of particles in the solution is higher and therefore the particles are shading each other to a greater extent (Fig. 4, left side)), the total surface area that is offered is larger and the sum of cathodic (dark) currents is higher. This is shown schematically in Fig. 4, right side. (The experimentally observed current under illumination (not shown for clarity) is the sum of the photocurrent and the dark current). If a given number of photons is shared by two particles instead of one, each of them will produce half of the photocurrent, $i_{\mathrm{ph}} / 2$, but will offer the full dark current, $i_{\mathrm{d}}$, corresponding to its surface area (Fig. 4, right side). A smaller photocurrent means a working potential being father away from the photocurrent onset potential. By summing up the local cell currents at the intersection, we see that $2 i_{\mathrm{SC} 2}>i_{\mathrm{SC} 1}$, which means that a higher total current is drawn when the same amount of light is shared by two particles. The situation is even more favourable if more than two particles are involved. ${ }^{[7]}$ However, this effect is ultimately limited by the onset potential of the dark currents. As shown in the beginning, there are ways of shifting this onset potential (for instance by metallization). However, no working potential above this onset potential is accessible without the help of external bias, which can not be applied for particle suspensions or particles immobilized on non-conducting surfaces.

Even in the case of external bias (immobilized catalyst) where the working point of the photoelectrode can be set in a potential region where plateau currents flow, there
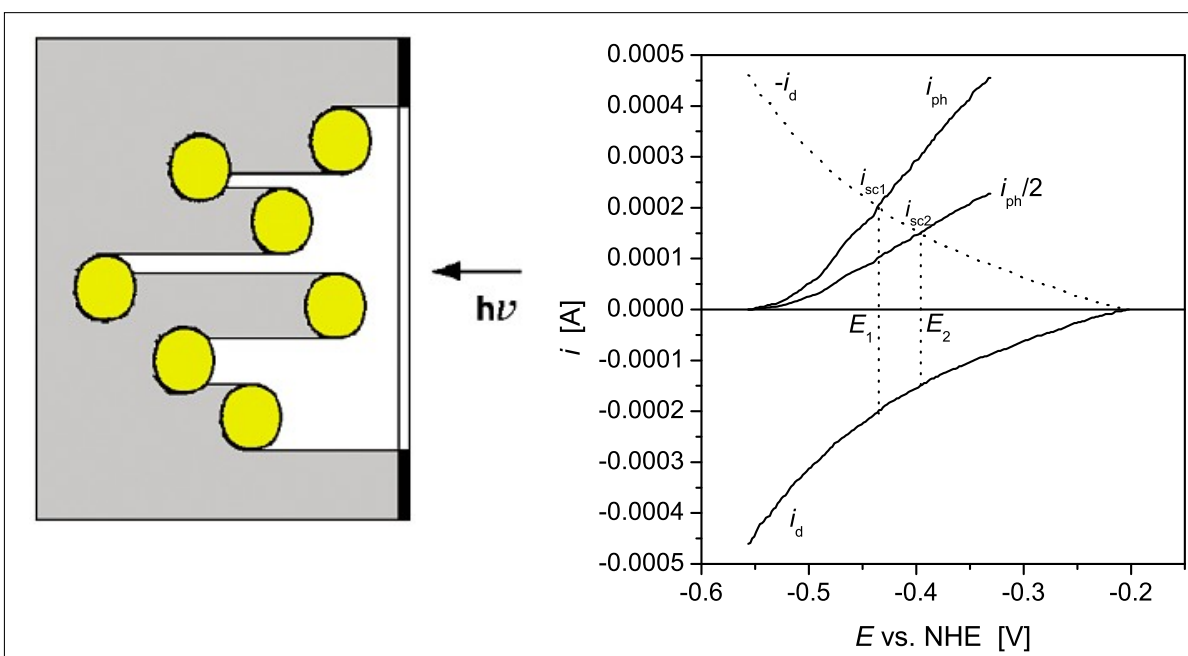

Fig. 4. Sharing of a given number of photons by several particles suspended in solution. Left: illumination of a particle suspension through a mask (schematic), right: $i-E$ curves for light being absorbed by one or two particles. $i_{\mathrm{ph}}=i_{\text {light }}-i_{\mathrm{d}}$.

can be a benefit of large surface area (again considering the same given overall geometrical area of the cell). If the surface is rough, for the same total photocurrent as produced by a smooth surface, the photocurrent density will be smaller. If the oxidation of the donor (the organic molecule that is to be oxidatively destroyed) reaction proceeds (partly) via $\mathrm{OH}$ radicals, their recombination represents a 'chemical short circuit', and this recombination will be less efficient at low current densities, as the rate of this bimolecular reaction depends on the square of the local radical concentrations. ${ }^{[10]}$

\section{Nanoparticulate Electrodes}

A special case are nanoparticulate electrodes, i.e. electrodes obtained by packing together nanometre-sized catalyst particles like $\mathrm{TiO}_{2}$ P25 (Degussa) into a loosely agglomerated (porous) electrode. In this case, the electrolyte reaches up to the back contact of the electrode. Although there is no local field (no space charge region), majority carriers are removed by diffusion and reaction (oxidation of the donor) takes place all over the porous structure. Only a small electrical bias is needed for extracting the majority carriers at the back contact. However, although quite promising, so far such electrodes have not shown the stability required for process implementation.

\section{Conclusion}

An overview is given of the essential processes governing heterogeneous photocatalysis on semiconductor surfaces. It is shown that the reactions on the surface of the catalyst are of electrochemical nature and can be described using electrochemical principles. This leads to a quantitative de- scription of the processes. In combination with measurements of charge collection yields and Faradaic efficiencies, or product quantum yields, the reaction rates can be calculated when the spectral dependence of the intensity of the light source is known. Examples are given for transition metal oxide semiconductors such as titanium dioxide and tungsten trioxide. Considering the rectifying nature of semiconductor oxide/ aqueous electrolyte junctions, it follows that electrical bias can boost the photocatalytic performance considerably. In a batch reactor, under bias and, the degradation of a donor follows an exponential decrease and is proportional to the light intensity if sufficient mass transport (liquid flow) is provided.

Received: October 17, 2007

[1] G. Ruppert, R. Bauer, G. Heisler, S. Novalic, Chemosphere 1993, 27, 1339.

[2] A. Mills, S. Le Hunte, J. Photochem. Photobiol. 1997, 108, 1.

[3] O. M. Alfano, D. Bahnemann, A.E. Cassano, R. Dillert, R. Goslich, Catalysis Today 2000, 58, 199.

[4] J. Zhao, X. Yang, Building and Environment 2003, 38, 645.

[5] D. A. Tryk, A. Fujishima, 'The Electrochemical Society Interface', 2001, p. 32.

[6] M. Neumann-Spallart in 'Metallic Corrosion. Principles and Control', Eds. S. Khanna, S. N. Malhotra, K. S. V. Santhanam, M. K. Totlani, Wiley Eastern, New Delhi, 1994.

[7] M. Neumann-Spallart, O. Enea, $J$. Electrochem. Soc. 1984, 131, 2767.

[8] J. Desilvestro, M. Neumann-Spallart, $J$. Phys. Chem. 1985, 89, 3684.

[9] G. Waldner, A. Brüger, N. S. Gaikwad, M. Neumann-Spallart, Chemosphere 2007, 67,779 .

[10] G. Waldner, R. Gómez, M. NeumannSpallart, Electrochim. Acta 2007, 52, 2634. 